\title{
Simple and effective method for the analytic description of important optical beams when truncated by finite apertures
}

\author{
Michel Zamboni-Rached, ${ }^{1, *}$ Erasmo Recami, ${ }^{2,3,4,6}$ and Massimo Balma ${ }^{5}$ \\ 'DMO-FEEC, University of Campinas, Campinas, São Paulo, Brazil \\ ${ }^{2}$ Istituto Nazionale di Fisica Nucleare (INFN)-Sezione di Milano, Milan, Italy \\ ${ }^{3}$ Facoltà di Ingegneria, Università Statale di Bergamo, Bergamo, Italy \\ ${ }^{4}$ Departamento de Microonda e Óptica - Faculdade de Engenharia Elétrica e de Computação (DMO-FEEC), \\ Universidade Estadual de Campinas, São Paulo, Brazil \\ ${ }^{5}$ SELEX Galileo, San Maurizio Caselle Torino, Italy \\ ${ }^{6} e$-mail: recami@mi.infn.it \\ *Corresponding author: mzamboni@dmo.fee.unicamp.br \\ Received 8 March 2012; accepted 29 March 2012; \\ posted 3 April 2012 (Doc. ID 164377); published 30 May 2012

\begin{abstract}
In this paper we present a simple and effective method, based on appropriate superpositions of Bessel-Gauss beams, which in the Fresnel regime is able to describe in analytic form the threedimensional evolution of important waves as Bessel beams, plane waves, Gaussian beams, and Bessel-Gauss beams when truncated by finite apertures. One of the by-products of our mathematical method is that one can get in a few seconds, or minutes, high-precision results, which normally require quite lengthy numerical simulations. The method works in electromagnetism (optics, microwaves) as well as in acoustics. (C) 2012 Optical Society of America
\end{abstract} \\ OCIS codes: $\quad 260.1960,070.7345,110.1220,050.1755$.
}

\section{Introduction}

The analytic description of wave beam (in particular, optical beam) propagation is of high importance, both in theory and in practice. Because of the mathematical difficulties met when looking for exact solutions, often the analytic description must be obtained in an approximate way, and several other times one must have recourse to a numerical solution of the (differential or integral) propagation equations both in their exact or approximate forms.

A very common approximation is the paraxial one [1], rather useful for obtaining analytic or numerical solutions. For example, it is by such an approxi-

$1559-128 \mathrm{X} / 12 / 163370-10 \$ 15.00 / 0$

(ㄷ) 2012 Optical Society of America mation that one obtains the well-known Fresnel diffraction integral [1], which yields accurate (analytic and numerical) results for a large part of the proximal field region, as well as in the transition region toward the distant field.

An important analytic solution forwarded by the Fresnel diffraction integral is the Gaussian beam, while another one is the Bessel-Gauss beam, found by Gori and Guattari [2] in 1987. The latter, endowed with a transverse profile in which the Bessel function is modulated by a Gaussian function, can be regarded as an experimentally realizable version of the Bessel beam; indeed, the Bessel beam is a quite noticeable exact, nondiffracting solution of the wave equation, but is associated with an infinite power flux (through any plane orthogonal to the 
propagation axis), as also happens, for instance, with plane waves.

Notwithstanding the fact that some analytic solutions do exist for the Fresnel diffraction integral, they are rare, and normally it is necessary to have recourse to numerical simulations. This is particularly true when the mentioned integral is adopted for the description of beams generated by finite apertures, that is, of beams truncated in space.

The past attempts at an analytic description of truncated beams were based on a Fresnel integral: probably the best known of them being the Wen and Breazele method [3], using superpositions of Gaussian beams (with different waist sizes and positions) in order to describe axially symmetrical beams truncated by circular apertures. In that approach, those authors had to adopt a computational optimization process to get the superposition coefficients and the beam waists and spot positions of the various Gaussian beams; actually, the necessity of a computational optimization to find out which beam superposition is adequate to describe a certain truncated beam is due to the simple fact that the Gaussian beams do not constitute an orthogonal basis.

To cope with the difficulty related with such a computational optimization, Ding and Zhang [4] modified the method by choosing since the beginning the beam waist values and then writing down a set of linear equations in terms of the Gaussian beam superposition coefficients. However, in that new approach the nonhomogeneous terms are given by integrals that, once more, cannot in general be easily evaluated in closed form.

In this paper we show that an analytic description of important truncated beams can be obtained by means of Bessel-Gauss beam superpositions, whose coefficients are obtained in a simple and direct way, without any need of numerical optimizations or of equation system solutions.

Indeed, our method is capable of yielding analytic solutions for the three-dimensional evolution of Bessel beams, plane waves, Gaussian beams, and Bessel-Gauss beams, when truncated by finite apertures in the Fresnel regime.

\section{Fresnel Diffraction Integral and Some Solutions}

For simplicity's sake, we shall leave understood in all solutions the harmonic time-dependence term $\exp (-i \omega t)$.

In the paraxial approximation, an axially symmetric monochromatic wave field can be evaluated, knowing its shape on the $z=0$ plane, through the Fresnel diffraction integral in cylindrical coordinates:

$$
\begin{aligned}
\Psi(\rho, z)= & \frac{-i k}{z} \exp \left[i\left(k z+\frac{k \rho^{2}}{2 z}\right)\right] \int_{0}^{\infty} \Psi\left(\rho^{\prime}, 0\right) \\
& \times \exp \left(i k \frac{\rho^{\prime 2}}{2 z}\right) J_{0}\left(k \frac{\rho \rho^{\prime}}{z}\right) \rho^{\prime} \mathrm{d} \rho^{\prime}
\end{aligned}
$$

where $k=2 \pi / \lambda$ is the wavenumber, and $\lambda$ the wavelength. In this equation, $\rho^{\prime}$ reminds us that the integration is being performed on the plane $z=0$; thus, $\Psi\left(\rho^{\prime}, 0\right)$ does simply indicate the field value on $z=0$.

Let us consider a Gaussian behavior on $z=0$; that is, let us choose the "excitation"

$$
\Psi\left(\rho^{\prime}, 0\right)=\Psi_{G}\left(\rho^{\prime}, 0\right)=A \exp \left(-q \rho^{\prime 2}\right),
$$

contained in [1]; with $A$ and $q$ being constants that can have complex values. For $\operatorname{Re}(q) \geq 0$, one gets the well-known Gaussian beam solution:

$$
\Psi_{G}(\rho, z)=-\frac{i k A}{2 z Q} \exp \left[i k\left(z+\frac{\rho^{2}}{2 z}\right)\right] \exp \left[-\frac{k^{2} \rho^{2}}{4 Q z^{2}}\right]
$$

where

$$
Q=q-i k / 2 z
$$

Another important solution is obtained by considering on the $z=0$ plane the excitation given by

$$
\Psi\left(\rho^{\prime}, 0\right)=\Psi_{\mathrm{BG}}\left(\rho^{\prime}, 0\right)=A J_{0}\left(k_{\rho} \rho^{\prime}\right) \exp \left(-q \rho^{\prime 2}\right),
$$

which, according to Eq (1), produces the so-called Bessel-Gauss beam [2]:

$$
\begin{aligned}
\Psi_{\mathrm{BG}}(\rho, z)= & -\frac{i k A}{2 z Q} \exp \left[i k\left(z+\frac{\rho^{2}}{2 z}\right)\right] J_{0}\left(\frac{i k k_{\rho} \rho}{2 z Q}\right) \\
& \times \exp \left[-\frac{1}{4 Q}\left(k_{\rho}^{2}+\frac{k^{2} \rho^{2}}{z^{2}}\right)\right],
\end{aligned}
$$

with quantity $Q$ being given by Eq. ( $\underline{4})$ and $k_{\rho}$ being a constant. (Quantity $k_{p}$ is the transverse wavenumber associated with the Bessel beam transversally modulated by the Gaussian function.)

The Bessel-Gauss beam given by Eq. (6) is particularly interesting since it can well be regarded as a realistic version (experimentally speaking) of the ideal Bessel beam:

$$
\Psi_{B}(\rho, z)=A J_{0}\left(k_{\rho} \rho\right) \exp \left(i k_{z} z\right)
$$

where $k_{z}=\sqrt{\omega^{2} / c^{2}-k_{\rho}^{2}}$.

The Bessel beam, Eq. (7), is an exact solution to the wave equation and is known to possess the important characteristic of keeping its transverse behavior unchanged while propagating, so it belongs to the class of nondiffracting beams. However, the ideal Bessel beam is endowed with an infinite power flux, and cannot be concretely generated. By contrast, the Bessel-Gauss beam, Eq. (ㅁ), modulates in space the transverse behavior of the Bessel beam by a Gaussian function, getting a finite power flux. The Bessel-Gauss beam will no longer remain 
indefinitely undistorted, but nevertheless shows to possess a rather good resistance to diffraction [2].

The Gaussian beam, Eq. (3), and the BesselGauss, Eq. (6), solutions are among the few solutions to the Fresnel diffraction integral that can be had analytically. The situation gets much more complicated, however, when facing beams truncated in space by finite circular apertures: for instance, a Gaussian beam, a Bessel beam, or a Bessel-Gauss beam, truncated via an aperture with radius $R$. In this case, the upper limit of the integral in Eq. (1) becomes the aperture radius, and the analytic integration becomes very difficult, requiring recourse to lengthy numerical calculations.

As we have already mentioned, in their alternative attempt at describing truncated beams, Wen and Breazele [3] adopted superpositions of Gaussian beams. To be, now, more specific, those authors gave the solution for a wave equation in the paraxial approximation as

$\Psi(\rho, z)=-\frac{i k}{2 z} \exp \left[i k\left(z+\frac{\rho^{2}}{2 z}\right)\right] \sum_{n=1}^{N} \frac{A_{n}}{Q_{n}} \exp \left[-\frac{k^{2} \rho^{2}}{4 Q_{n} z^{2}}\right]$,

with

$$
Q_{n}=q_{n}-\frac{i k}{2 z}
$$

Solution (8) is a superposition of $N$ Gaussian beams. Its coefficients $A_{n}$ and $q_{n}$ are to be obtained starting from the field existing on the $z=0$ plane, that is, starting from the initial excitation that we shall call $V(\rho)$. One therefore looks for $\Psi(\rho, 0)=V(\rho)$. From Eq. () one gets

$$
V(\rho)=\sum_{n=1}^{N} A_{n} \exp \left(-q_{n} \rho^{2}\right) .
$$

The initial field $V(\rho)$ can represent a beam (e.g., a plane wave, a Gaussian beam, etc.) truncated by a circular aperture with radius $R$. To get the coefficients $A_{n}$ and $q_{n}$ from Eq. (10), those authors had recourse to a computational optimization process in order to minimize the mean square error: Namely, to minimize the difference between the desired function $V(\rho)$ and the Gaussian series in the right-hand side (r.h.s.) of Eq. (10). Such a method yields good results, provided that the excitation function $V(\rho)$ does not oscillate too much. But the coefficients $A_{n}$ and $q_{n}$ are obtained in a strictly nonalgebraic manner, depending on the contrary on numerical calculations.

Let us be more specific also about the modification of Wen and Breazele's method introduced by Ding and Zhang [4]. They postulated the values of the parameters $q_{n}$ and, by minimizing the mean square error between the desired function $V(\rho)$ and the Gaussian series in Eq. (10), arrived at a system of linear equations containing the unknowns $A_{n}$ (the coefficients of the Gaussian beam superposition), without needing a numerical optimization process.
However, in the system of equations needed to determine the coefficients $A_{n}$, the nonhomogeneous terms consist in integrals that, depending on the field one wishes to truncate [i.e., depending on $V(\rho)$ ], can be difficult to calculate analytically.

In the next section we are going to propose a method, for the description of truncated beams, that appears to be noticeable for its simplicity and, in most cases, for its total analyticity. Our method is based on Bessel-Gauss beam superpositions, the coefficients of which can be directly evaluated without any need of computational optimizations or the solving of any coupled equation systems.

\section{Method}

Let us start with the Bessel-Gauss beam solution, Eq. ( $\underline{6})$ and consider the solution given by the following superposition of such beams:

$$
\begin{aligned}
\Psi(\rho, z)= & -\frac{i k}{2 z} \exp \left[i k\left(z+\frac{\rho^{2}}{2 z}\right)\right] \sum_{n=-N}^{N} \frac{A_{n}}{Q_{n}} J_{0}\left(\frac{i k k_{\rho} \rho}{2 z Q_{n}}\right) \\
& \times \exp \left[-\frac{1}{4 Q_{n}}\left(k_{\rho}^{2}+\frac{k^{2} \rho^{2}}{z^{2}}\right)\right],
\end{aligned}
$$

with the quantities $A_{n}$ being constants and $Q_{n}$ being given by Eq. (9), so that $Q_{n}=q_{n}-i k / 2 z$, where $q_{n}$ are constants that can have complex values. Notice that in this superposition, all beams possess the same value of $k_{\rho}$.

Let us recall, incidentally, that all the beams we are considering in this work are important particular cases of the so-called localized waves (see [ $[\underline{5},-\underline{7}]$ and references therein; see also $[\underline{8}, \underline{9}]$ ).

Our purpose is that solution (11) be able to represent beams truncated by circular apertures: as announced, we are particularly interested in the analytic description of truncated beams of Bessel, Bessel-Gauss, Gaussian, and plane wave types.

Given one of such beams truncated at $z=0$ by an aperture with radius $R$, we have to determine the coefficients $A_{n}$ and $q_{n}$ in such a way that Eq. (11) represents with fidelity the resulting beam. If the truncated beam on the $z=0$ plane is given by $V(\rho)$, we have to obtain $\Psi(\rho, 0)=V(\rho)$; that is,

$$
V(\rho)=J_{0}\left(k_{\rho} \rho\right) \sum_{n=-N}^{N} A_{n} e^{-q_{n} \rho^{2}} .
$$

The r.h.s. of this equation is nothing but a superposition of Bessel-Gauss beams, all with the same value $k_{\rho}$, at $z=0$ [namely, each one of such beams is written at $z=0$ according to Eq. (5)].

Equation (12) will provide us with the values of the $A_{n}$ and $q_{n}$, as well as of $N$. Once these values have been obtained, the field emanated by the finite circular aperture located at $z=0$ will be given by Eq. (11). Remembering that the $q_{n}$ can be complex, let us make the following choices: 


$$
q_{n}=q_{R}+i q_{\mathrm{In}},
$$

where $q_{R}>0$ is the real part of $q_{n}$, having the same value for every $n$, and $q_{\text {In }}$ is the imaginary part of $q_{n}$ given by

$$
q_{\text {In }}=-\frac{2 \pi}{L} n
$$

where $L$ is a constant with the dimensions of a square length.

With such choices, and assuming $N \rightarrow \infty$, Eq. (12) gets written as

$$
V(\rho)=J_{0}\left(k_{\rho} \rho\right) \exp \left(-q_{R} \rho^{2}\right) \sum_{n=-\infty}^{\infty} A_{n} \exp \left(i \frac{2 \pi n}{L} \rho^{2}\right),
$$

which then has to be exploited for obtaining the values of $A_{n}, k_{\rho}, q_{R}$, and $L$.

Let us recall that the aim of our method is describing some important truncated beams starting from the value of their near fields (i.e., of their fields in the Fresnel region).

In the cases of a truncated Bessel beam (TB) or of a truncated Bessel-Gauss beam (TBG), it seems natural to choose quantity $k_{\rho}$ in Eq. (15) to be equal to the corresponding beam transverse wavenumber.

In the case of a truncated Gaussian beam (TG) or of a truncated plane wave (TP), by contrast, it is natural to choose $k_{\rho}=0$ in Eq. (15).

In all cases, the product

$$
\exp \left(-q_{R} \rho^{2}\right) \sum_{n=-\infty}^{\infty} A_{n} \exp \left(i \frac{2 \pi n}{L} \rho^{2}\right),
$$

in Eq. (15) must represent:

(i) a function $\operatorname{circ}(\rho / R)$, in the TB or TP cases;

(ii) a function $\exp \left(-q \rho^{2}\right) \operatorname{circ}(\rho / R)$, that is, a circ function multiplied by a Gaussian function, in the TBG or TG cases.

Of course (i) is a particular case of (ii) with $q=0$. It may be useful to recall that the circ function is the step function in the cylindrically symmetrical case. Quantity $R$ is still the aperture radius, and $\operatorname{circ}(\rho / R)=1$ when $0 \leq \rho \leq R$, and it equals 0 in the contrary case.

Let us now show how Eq. (16) can approximately represent the above functions, given in (i) and (ii). Toward such an aim, let us consider a function $G(r)$ defined on an interval $|r| \leq L / 2$ and possessing the Fourier expansion:

$$
G(r)=\sum_{n=-\infty}^{\infty} A_{n} \exp (i 2 \pi n r / L) \quad \text { for }|r| \leq L / 2,
$$

where $r$ and $L$, having the dimensions of a square length, will be expressed in square meters $\left(\mathrm{m}^{2}\right)$.
Suppose now the function $G(r)$ is to be given by

$$
G(r)=\left\{\begin{array}{l}
\exp \left(q_{R} r\right) \exp (-q r) \quad \text { for }|r| \leq R^{2} \\
0 \quad \text { for } R^{2}<|r|<L / 2
\end{array}\right.
$$

where $q$ is a given constant. In this case, the coefficients $A_{n}$ in the Fourier expansion of $G(r)$ will be given by

$$
\begin{aligned}
A_{n}= & \frac{1}{L} \int_{-R^{2}}^{R^{2}} \exp \left(q_{R} r\right) \exp (-q r) \exp \left(-i \frac{2 \pi n}{L} r\right) \mathrm{d} r \\
= & \frac{1}{L\left(q_{R}-q\right)-i 2 \pi n}\left\{\exp \left[\left(q_{R}-q-i \frac{2 \pi}{L} n\right) R^{2}\right]\right. \\
& \left.-\exp \left[-\left(q_{R}-q-i \frac{2 \pi}{L} n\right) R^{2}\right]\right\} .
\end{aligned}
$$

Writing now

$$
r=\rho^{2},
$$

in Eqs. (17) and (18), we have

$$
\begin{aligned}
& \sum_{n=-\infty}^{\infty} A_{n} \exp \left(i 2 \pi n \rho^{2} / L\right) \\
& \quad=\left\{\begin{array}{l}
\exp \left(q_{R} \rho^{2}\right) \exp \left(-q \rho^{2}\right) \quad \text { for }|\rho| \leq R \\
0 \quad \text { for } R<|\rho|<\sqrt{L / 2},
\end{array}\right.
\end{aligned}
$$

where the coefficients $A_{n}$ are still given by Eq. (19). One recognizes that the left-hand side (l.h.s.) of Eq. (21) is the term multiplying the Gaussian in Eq. (16).

The 1.h.s. of Eq. (21), which depends on $\rho^{2}$, will not be exactly periodical: but it will reassume the values, assumed in the fundamental interval $(|\rho|<\sqrt{(L / 2)})$, in shorter and shorter further intervals, with decreasing spatial "periodicity," for $|\rho|>\sqrt{(L / 2)}$. In such a way, with $\rho \geq 0$, Eq. (16) becomes

$$
\begin{aligned}
& \exp \left(-q_{R} \rho^{2}\right) \sum_{n=-\infty}^{\infty} A_{n} \exp \left(i 2 \pi n \rho^{2} / L\right) \\
& \quad=\left\{\begin{array}{l}
\exp \left(-q \rho^{2}\right) \quad \text { for } 0 \leq \rho \leq R \\
0 \quad \text { for } R<\rho \leq \sqrt{L / 2} \\
\exp \left(-q_{R} \rho^{2}\right) f(\rho) \approx 0 \text { for } \rho>\sqrt{L / 2},
\end{array}\right.
\end{aligned}
$$

where $f(\rho)$ is a function existing on decreasing space intervals and assuming $\exp \left[\left(q_{R}-q\right) R^{2}\right]$ (if $q_{R}>q$ ) or 1 (if $q_{R} \leq q$ ) as its maximum values. Since $\sqrt{L / 2}>R$, for suitable choices of $q_{R}$ and $L$, we have $\exp \left(-q_{R} \rho^{2}\right) f(\rho) \approx 0$ for $\rho \geq \sqrt{L / 2}$.

Therefore, we get

$$
\begin{aligned}
& \exp \left(-q_{R} \rho^{2}\right) \sum_{n=-\infty}^{\infty} A_{n} \exp \left(i 2 \pi n \rho^{2} / L\right) \\
& \quad \approx \exp \left(-q \rho^{2}\right) \operatorname{circ}(\rho / R),
\end{aligned}
$$


which corresponds to case (i), when $q=0$, and to case (ii).

Let us recall once more that $A_{n}$ are given by Eqs. (19).

On the basis of what was shown before, we now have a very efficient method for describing important beams, truncated by finite apertures, namely, the TB, TG, TBG, and TP beams. Indeed, it is enough to choose the desired field, truncated by a circular aperture with radius $R$, and describe it at $z=0$ by our Eq. (15). Precisely:

- In the TBG case: the value of $k_{\rho}$ in Eq. (15) is the transverse wavenumber of the Bessel beam modulated by the Gaussian function, $A_{n}$ is given in Eq. (19), and $q$ is related to the Gaussian function width at $z=0$. The values $L$ and $q_{R}$, and the number $N$ of terms in the series (15), are chosen so as to guarantee a faithful description of the beam at $z=0$ when truncated by a circular aperture with radius $R$.

- In the TB case: the procedure will be the same as for TBG, but with $q=0$.

- In the TG case: the procedure is still the same as for TBG, but with $k_{\rho}=0$.

- In the TP case: the procedure is once more similar, but this time using $k_{\rho}=0$ and $q=0$.

Finally, once the chosen beam is described on the truncation plane $(z=0)$, the beam emanated by the finite aperture will be given by solution of Eq. (11).

It is important to notice at this point that, for a given truncation, innumerable sets of values of $q_{R}$ and $L$ exist that yield a faithful description of the truncated field. The choice is made in order that the solution given by the series (15) has good convergence properties. Of course, one will use a finite number $2 N+1$ of terms in the mentioned series, with $-N \leq n \leq N$. Let us go on to some examples.

\section{Applying the Method}

In this section we shall apply our method, as we already said, to situations in which important truncated beams appear. Notice that below we shall always assume a wavelength of $632.8 \mathrm{~nm}$.

\section{A. Analytic Description of the Truncated Bessel Beam}

Let us start from a Bessel beam, truncated at $z=0$ by a circular aperture with radius $R$; that is to say, from $\Psi_{\mathrm{TB}}(\rho, 0)=J_{0}\left(k_{\rho} \rho\right) \operatorname{circ}(\rho / R)$.

Let us choose $R=3.5 \mathrm{~mm}$ and the transverse wavenumber $k_{\rho}=4.07 \cdot 10^{4} \mathrm{~m}^{-1}$, which corresponds to a beam spot with radius approximatively equal to $\Delta \rho=59 \mu \mathrm{m}$ (while $\lambda=632.8 \mathrm{~nm}$, as always).

At $z=0$, the field is described by Eq. (15), where $A_{n}$ are given by Eq. (19) and where $q=0$. In this case, a quite good result can be obtained by the choice $L=3 R^{2}, q_{R}=6 / L$, and $N=23$. Let us repeat that, since such a choice is not unique, very many alternative sets of values $L$ and $q_{R}$ exist, which also yield excellent results.

Figure 1 shows the field given by Eq. (15): it represents with high fidelity the Bessel beam truncated at $z=0$.

The resulting field, emanated by the aperture, is given by solution (11), and its intensity is shown in Fig. 2. One can see that the result really corresponds to a Bessel beam truncated by a finite aperture. Figure 3 depicts the orthogonal projection of the same result.

Increasing $N$, that is, increasing the number of terms in the series (11), which expresses the resulting field, while keeping the same values for $L$ and $q_{R}$, the spatial shape of the obtained field practically will not change, but there will become more evident the rapid oscillations that occur at the beam crest, i.e., for $\Psi_{\mathrm{TB}}(\rho=0, z)$. This is shown in Fig. $\underline{4}$, where we used $N=500$.

\section{B. Analytic Description of the Truncated Gaussian Beam}

Let us go on now to consider a Gaussian beam truncated at $z=0$; that is, $\Psi_{\mathrm{TG}}(\rho, 0)=$ $\exp \left(-q \rho^{2}\right) \operatorname{circ}(\rho / R)$, whose initial intensity spot radius is $\Delta \rho=59 \mu \mathrm{m}$, and therefore $q=1 /\left(2 \Delta \rho^{2}\right)=$ $144 \cdot 10^{6} \mathrm{~m}^{-1}$. The radius of the circular aperture is

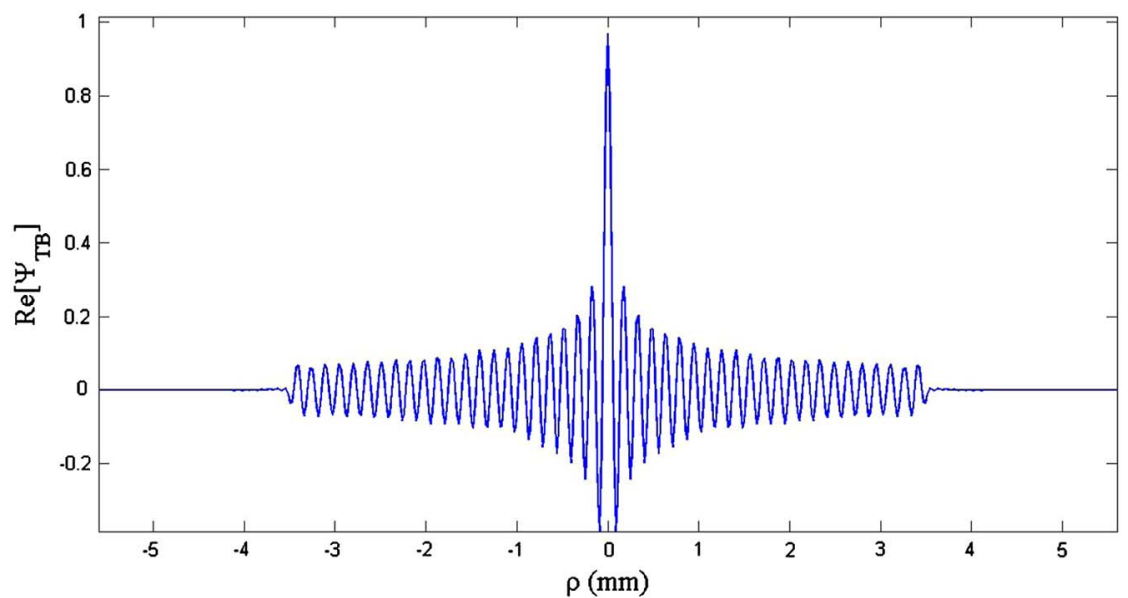

Fig. 1. (Color online) Field given by Eq. (15), representing a Bessel beam at $z=0$, with $k_{\rho}=4.07 \cdot 10^{4} \mathrm{~m}^{-1}$ and truncated by a finite circular aperture with radius $R=3.5 \mathrm{~mm}$. The coefficients $A_{n}$ are given by Eq. (19), with $q=0, L=3 R^{2}, q_{R}=6 / L$, and $N=23$. 


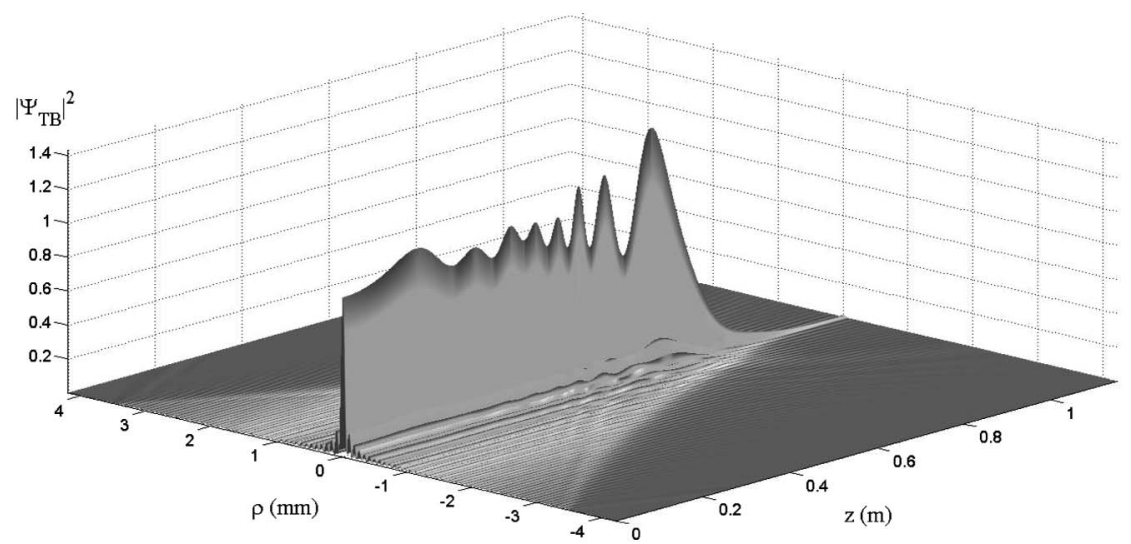

Fig. 2. Intensity of a Bessel beam truncated by a finite aperture, as given by solution (11).

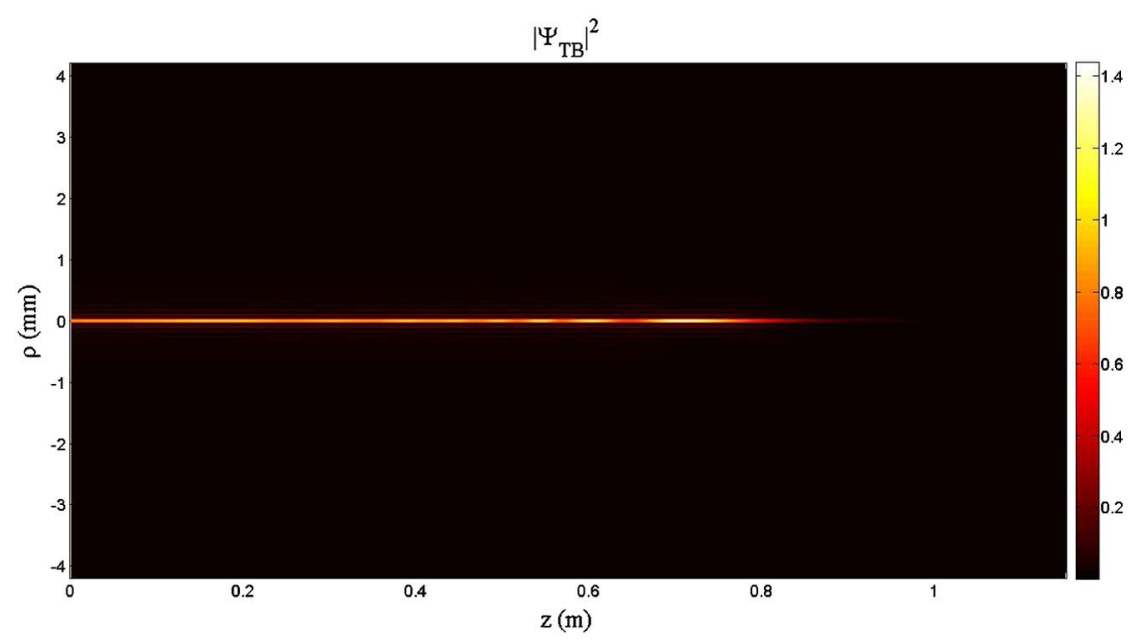

Fig. 3. (Color online) Orthogonal projection of the intensity shown in Fig. 2.

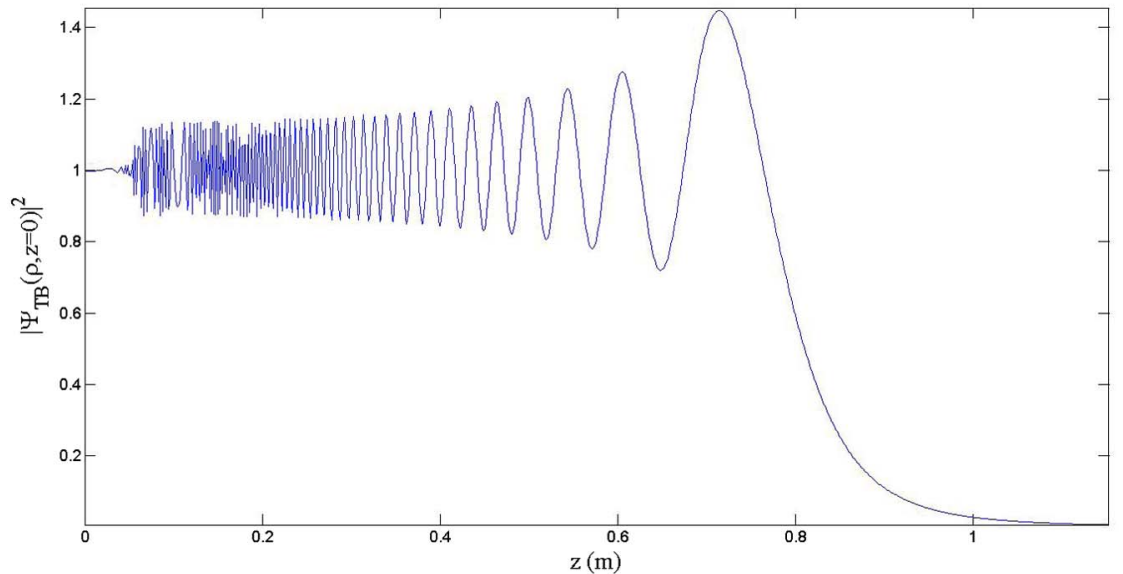

Fig. 4. (Color online) Oscillations of the field intensity on the $z$ axis, when adopting $N=500$ in Eq. (11).

equal to the beam spot radius, i.e., $R=59 \mu \mathrm{m}$, while, as always, $\lambda=632.8 \mathrm{~nm}$.

The situation at $z=0$ is still described by Eq. (15), with $k_{\rho}=0$, where $A_{n}$ are given by Eq. (19). Now, a good result can be obtained, for instance, by using the values $L=4 R^{2}, q_{R}=8 / L$, and $N=81$.
In Fig. 5 we show the field given by Eq. (15), in the case of a Gaussian beam truncated at $z=0$. The dotted line depicts the ideal Gaussian curve, without truncation.

The resulting field, emanated by the finite aperture, is given by the solution (11), and Fig. 6 shows 


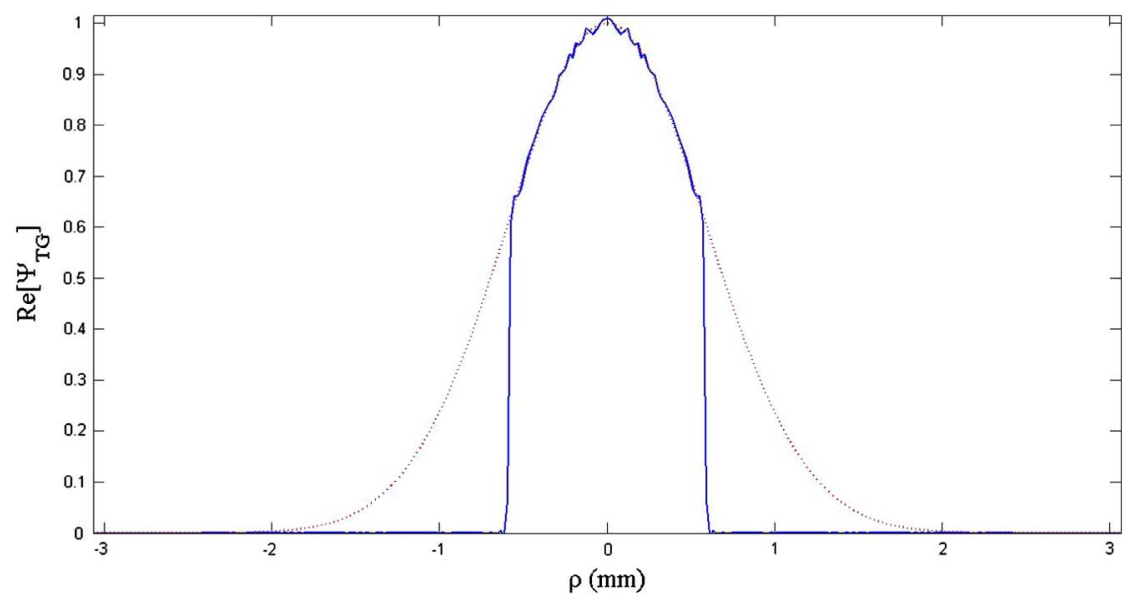

Fig. 5. (Color online) Field given by Eq. (15), when representing a Gaussian beam truncated at $z=0$. The dotted line depicts the ideal Gaussian curve, when truncation is absent.

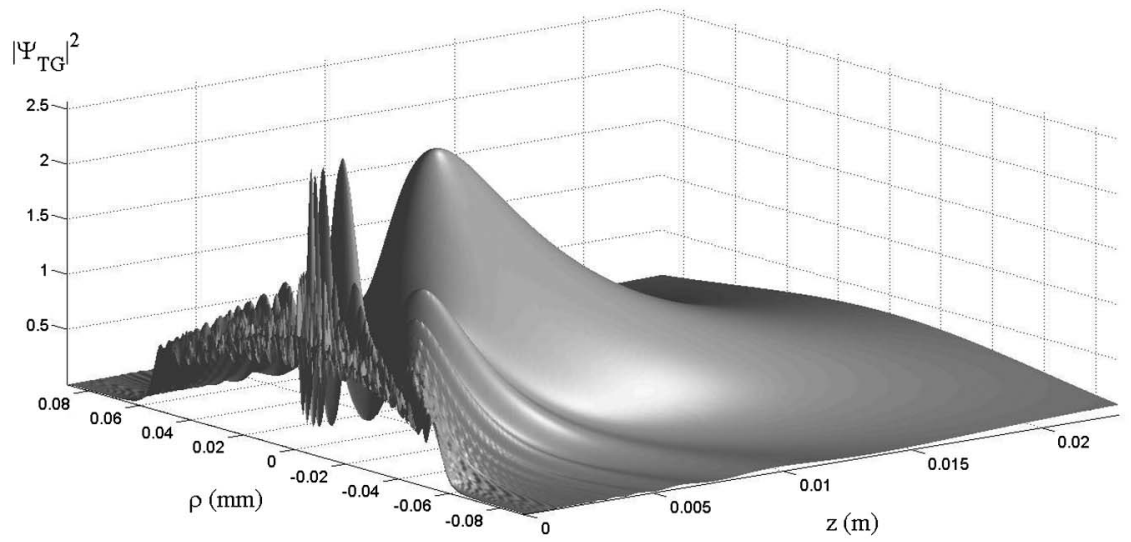

Fig. 6. Square magnitude of the field emanated by a finite aperture, in the case of a Gaussian beam, according to the solution (11).

its square magnitude. In Fig. $\underline{7}$ we show the corresponding orthogonal projection.

\section{Analytic Description of a Truncated Bessel-Gauss Beam}

Let us now consider the interesting case of a Bessel-Gauss beam truncated by a circular aperture of radius $R$; that is, $\Psi_{\mathrm{TBG}}(\rho, 0)=$ $J_{0}\left(k_{\rho} \rho\right) \exp \left(-q \rho^{2}\right) \operatorname{circ}(\rho / R)$, with $k_{\rho}=4.07 \cdot 10^{4} \mathrm{~m}^{-1}$, $q=1.44 \cdot 10^{6} \mathrm{~m}^{-1}, R=1 \mathrm{~mm}$, and $\lambda=632.8 \mathrm{~nm}$.

The situation at $z=0$ is described by Eq. (15), where $A_{n}$ are given by relations (19). A very good result can be obtained, e.g., by adopting the values $L=10 R^{2}, q_{R}=q$, and $N=30$. Figure 8 shows the field in Eq. (15), in the present case of a BesselGauss beam truncated at $z=0$.

The resulting field emanated by the finite aperture is given by solution (11), and Fig. 9 shows its square magnitude. Figure $1 \overline{0}$ depicts the orthogonal projection for this case.

\section{Analytic Solution of a Truncated Plane Wave}

Consider now the case of a plane wave truncated by a circular aperture at $z=0$, that is, $\Psi_{\mathrm{TP}}(\rho, 0)=$ $\operatorname{circ}(\rho / R)$, where we choose $R=1 \mathrm{~mm}$ and $\lambda=632.8 \mathrm{~nm}$.

Once more, Eq. (15) describes the field at $z=0$, with $k_{\rho}=0$, the coefficients $A_{n}$ being given by

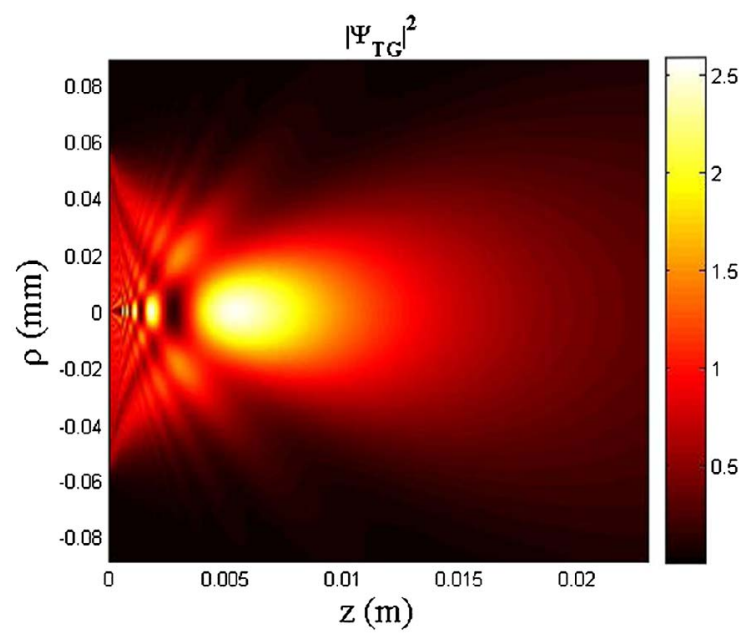

Fig. 7. (Color online) Orthogonal projection corresponding to Fig. 6 . 


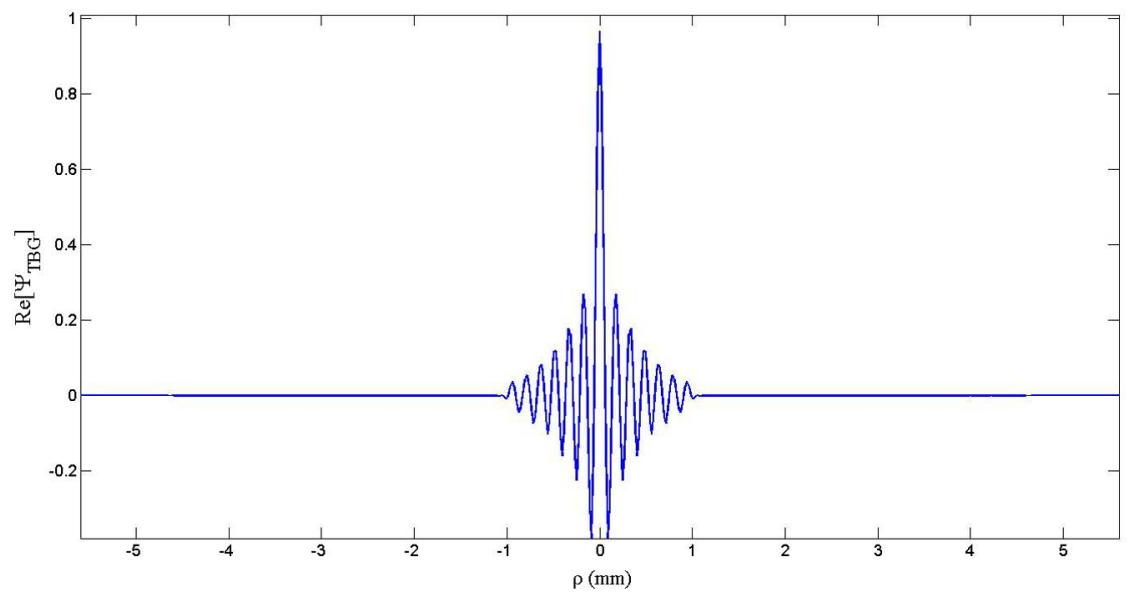

Fig. 8. (Color online) Field in Eq. (15), in the case now of a Bessel-Gauss beam truncated at $z=0$. Here we adopted the values $L=10 R^{2}$, $q_{R}=q$, and $N=30$; see the text for details.

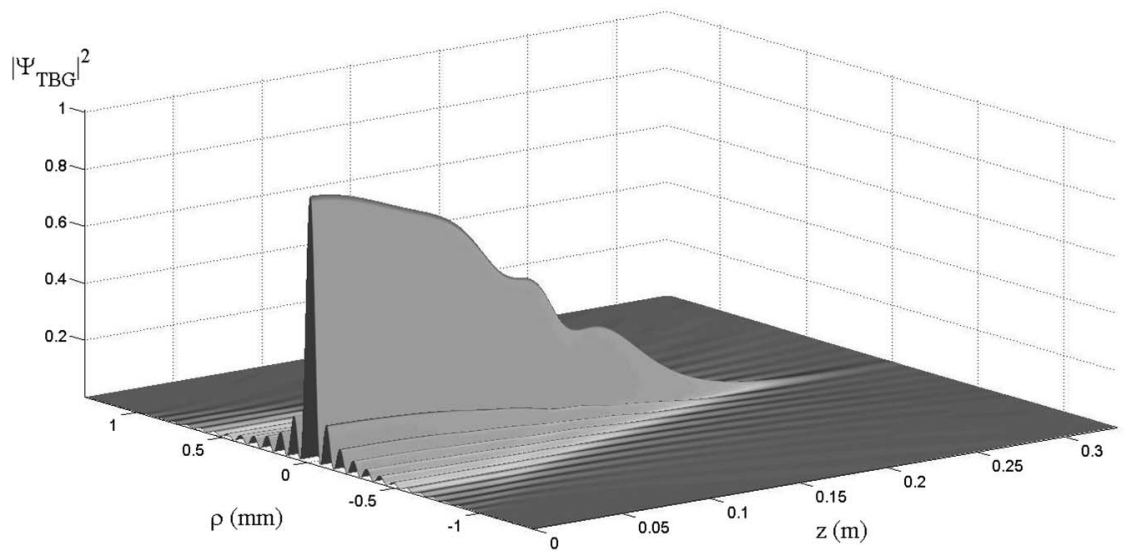

Fig. 9. Square magnitude of the field emanated by a finite aperture in the case of a truncated Bessel-Gauss beam, represented by solution (11).

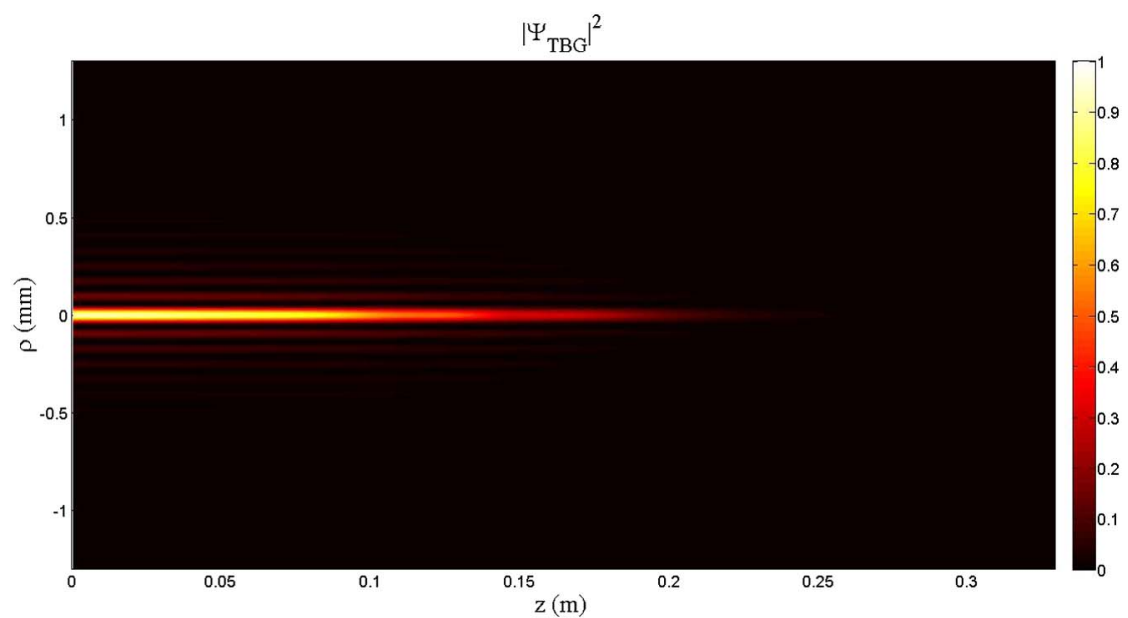

Fig. 10. (Color online) Orthogonal projection for the case in Fig. $\underline{9}$.

relations (19), with $q=0$. A good result can be obtained by adopting, e.g., the values $L=6 R^{2}$, $q_{R}=8 / L$, and $N=150$.

Figure 11 shows the field in Eq. (15), in the present case of a plane wave truncated at $\bar{z}=0$.
The resulting field emanated by the finite aperture is given by solution (11), and its square magnitude is shown in Fig. 12 . Figure $\underline{13}$ depicts the corresponding orthogonal projection. 


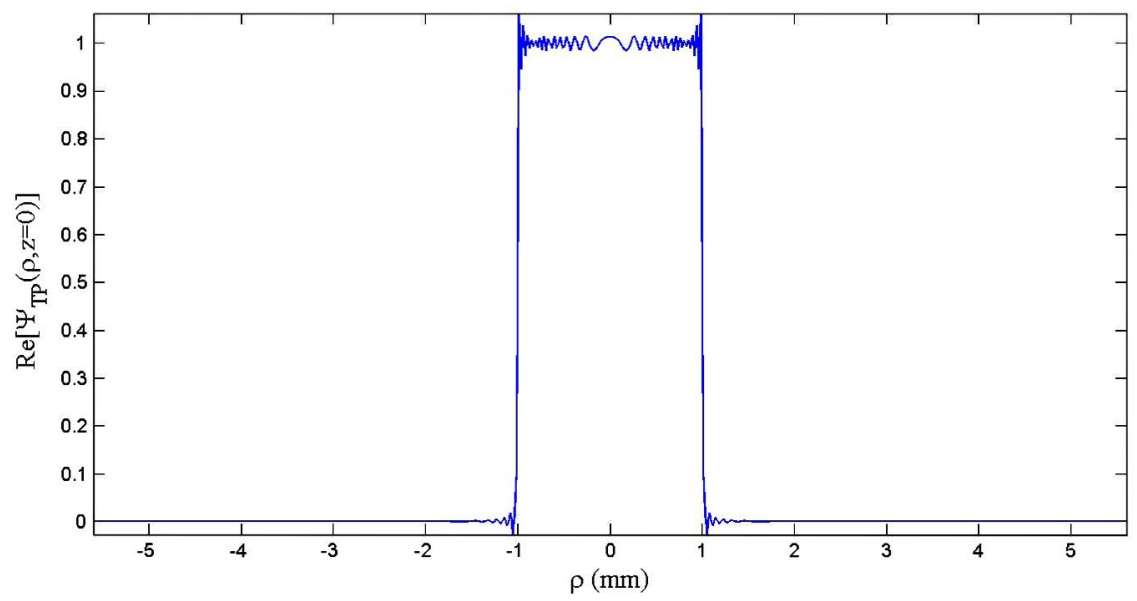

Fig. 11. (Color online) Field at $z=0$, with $k_{\rho}=0$, as given by Eq. (15) in the new case of a plane wave truncated at $z=0$. We have here adopted the values $L=6 R^{2}, q_{R}=8 / L$, and $N=150$. The coefficients $A_{n}$ being given by relations (19), with $q=0$.

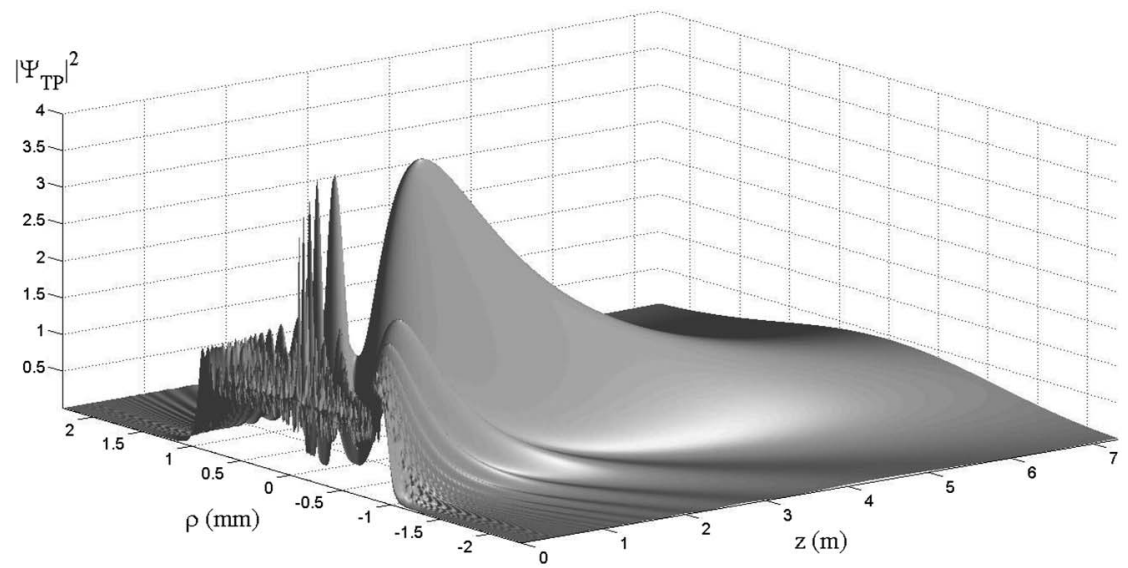

Fig. 12. Square magnitude of the resulting field, emanated by the finite aperture, as given by solution (11) in the present case of a truncated plane wave.

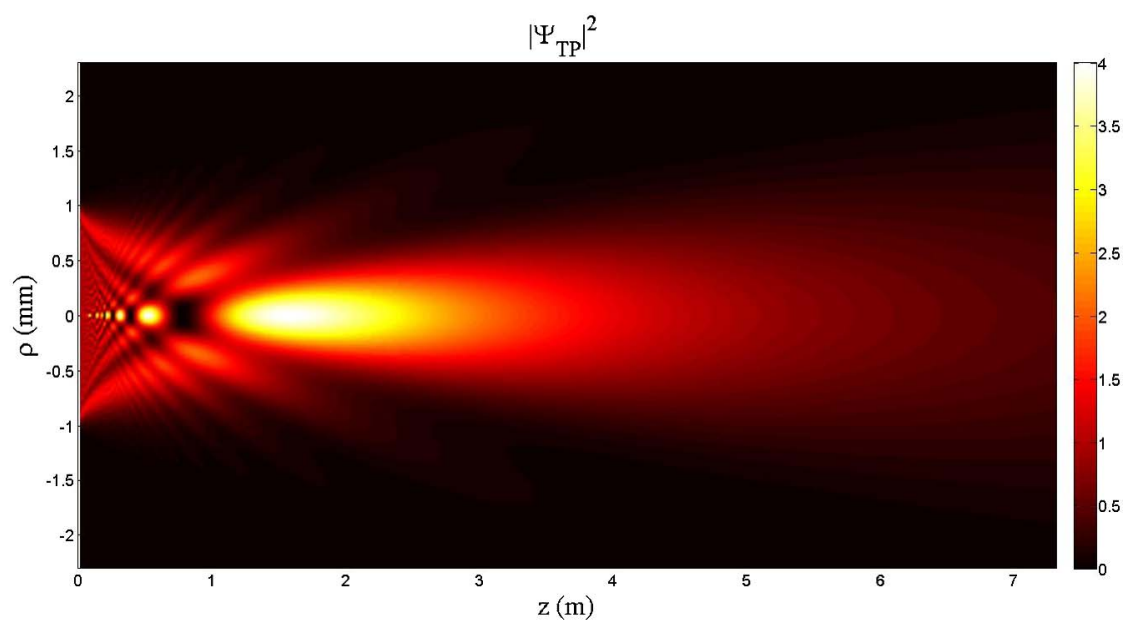

Fig. 13. (Color online) Orthogonal projection corresponding to Fig. 12.

\section{Conclusions}

In this paper, starting from suitable superpositions of Bessel-Gauss beams [2], we have constructed a simple and effective method for the analytic description, in the Fresnel region, of important beams truncated by finite apertures. 
The solutions obtained by our method, and representing truncated Bessel beams, truncated Gaussian beams, truncated Bessel-Gauss beams, and truncated plane waves, fully agree with the known results obtained by lengthy numerical evaluations of the corresponding Fresnel diffraction integrals. (Incidentally, let us mention that all the beams considered in this work are important particular cases of the so-called localized waves [5-8].)

At variance with the previous Wen and Breazele approach [3] (which uses a computational method of numerical optimization to obtain Gaussian beam superpositions describing truncated beams) and even at variance with the Ding and Zhang approach [4] (which is an improved version of [3]), our method does not need any numerical optimizations nor the numerical solution of any coupled equation systems.

Indeed, the simpler method exploited in this paper is totally analytic, and it directly applies to the beams considered above as well as to many other beams that are being investigated and will be presented elsewhere, such as truncated higher order Bessel and Bessel-Gauss beams, beams truncated by annular apertures, or beams truncated and modulated by convergent/divergent lenses. In particular, we have applied this method to remote sensing by microwaves (see, e.g., [10]), constructing finite antennas which emit truncated Bessel beams with the required characteristics. Of course, this method works in electromagnetism (optics, microwaves) as well as in acoustics.

Let us stress that one of the main by-products of our mathematical method is that by its use, one can get in a few seconds, or minutes, high-precision results that could otherwise require lengthy numerical simulation.

The authors are grateful to Giuseppe Battistoni, Carlos Castro, Mário Novello, Jane M. Madureira Rached, Nelson Pinto, Alberto Santambrogio, Marisa
Tenório de Vasconselos, and particularly Hugo E. Hernández-Figueroa for many stimulating contacts and discussions. The authors acknowledge partial support from the Fundação de Amparo à Pesquisa do Estado de São Paulo (under grant 11/51200-4), from the Conselho Nacional de Desenvolvimento Científico e Tecnológico (under grant 307962/20105 ), and one of us (E. Recami) acknowledges a past Coordenação de Aperfeiçoamento de Pessoal de Nível Superior fellowship from the Universidade Estadual de Campinas/Faculdade de Engenharia Elétrica e de Computação/Departamento de Microonda e Óptica.

\section{References}

1. J. W. Goodman, Introduction to Fourier Optics (McGraw-Hill, 1996).

2. F. Gori and G. Guattari, "Bessel-Gauss beams," Opt. Commun. 64, 491-495 (1987).

3. J. J. Wen and M. A. Breazele, "A diffraction beam field expressed as the superposition of Gaussian beams," J. Acoust. Soc. Am. 83, 1752-1756 (1988).

4. D. Ding and Y. Zhang, "Notes on the Gaussian beam expansion," J. Acoust. Soc. Am. 116, 1401-1405 (2004).

5. H. E. H. Figueroa, M. Z. Rached, and E. Recami, eds., Localized Waves (Wiley, 2008).

6. E. Recami and M. Z. Rached, "Localized waves: a review," in Advances in Imaging and Electron Physics, Vol. 156 (Academic, 2009), pp. 235-353.

7. E. Recami, M. Z. Rached, K. Z. Nóbrega, C. A. Dartora, and H. E. H. Figueroa, "On the localized superluminal solutions to the Maxwell equations," IEEE J. Sel. Top. Quantum Electron. 9, 59-73 (2003), special issue on "Nontraditional Forms of Light."

8. M. Z. Rached, "Unidirectional decomposition method for obtaining exact localized wave solutions totally free of backward components," Phys. Rev. A 79, 013816 (2009).

9. C. A. Dartora and K. Z. Nobrega, "Study of Gaussian and Bessel beam propagation using a new analytic approach," Opt. Commun. 285, 510-516 (2012).

10. M. Z. Rached, E. Recami, and M. Balma, "Proposte di antenne generatrici di fasci non-diffrattivi di microonde (Proposal of apertures generating nondiffracting beams of microwaves)," http://arxiv.org/abs/1108.2027. 